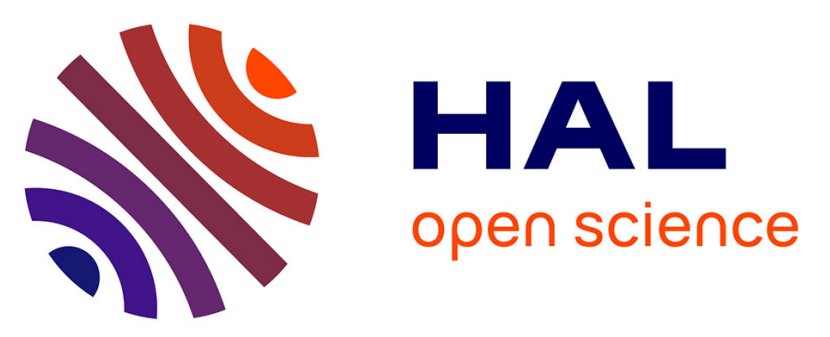

\title{
Le bien-être psychologique au travail des enseignants stagiaires en Éducation Physique et Sportive: une histoire de valeur
}

Clément Llena, Isabelle Joing, Jacques Mikulovic

\section{To cite this version:}

Clément Llena, Isabelle Joing, Jacques Mikulovic. Le bien-être psychologique au travail des enseignants stagiaires en Éducation Physique et Sportive: une histoire de valeur. Eduquer, 2020, Recherches \& éducations, 10.4000/rechercheseducations.8032 . hal-02525864v2

\section{HAL Id: hal-02525864 \\ https://hal.univ-lille.fr/hal-02525864v2}

Submitted on 7 Jun 2021

HAL is a multi-disciplinary open access archive for the deposit and dissemination of scientific research documents, whether they are published or not. The documents may come from teaching and research institutions in France or abroad, or from public or private research centers.
L'archive ouverte pluridisciplinaire HAL, est destinée au dépôt et à la diffusion de documents scientifiques de niveau recherche, publiés ou non, émanant des établissements d'enseignement et de recherche français ou étrangers, des laboratoires publics ou privés. 


\title{
Le bien-être psychologique au travail des enseignants stagiaires en Éducation Physique et Sportive : une histoire de valeurs
}

\author{
Univ. Lille, Univ. Littoral Côte d'Opale, Univ. Artois, EA 7369 - URePSSS - Unité de Recherche \\ Pluridisciplinaire Sport Santé Société, F-59000 Lille, France \\ Univ. Bordeaux, LACES - Laboratoire Cultures Éducation Sociétés - EA 7437, F-33000 Bordeaux \\ Isabelle Joing \\ Univ. Lille, Univ. Littoral Côte d'Opale, Univ. Artois, EA 7369 - URePSSS - Unité de Recherche \\ Pluridisciplinaire Sport Santé Société, F-59000 Lille, France
}

\section{Jacques Mikulovic}

Univ. Bordeaux, LACES - Laboratoire Cultures Éducation Sociétés - EA 7437, F-33000 Bordeaux INSHEA, Grhapes - Groupe de recherche sur le handicap, l'accessibilité, les pratiques éducatives et scolaires - EA 7287, F-92150 Suresnes, France

\begin{abstract}
Résumé : L'objet de cet article est d'examiner le lien entre les systèmes de valeurs des enseignants stagiaires en Éducation Physique et Sportive (EPS) et leur niveau de bien-être psychologique au travail (BEPT). L'hypothèse principale postule que les enseignants qui valorisent les valeurs d'ouverture au changement et de dépassement de soi ont un niveau de BEPT plus élevé. Au contraire les enseignants qui sont tournés vers des valeurs de continuité ressentent comparativement un bien-être au travail moins élevé. Une enquête empirique a été menée auprès de 184 enseignants stagiaires en EPS (58 femmes et 126 hommes) issus de 28 universités différentes en France. Les résultats valident l'hypothèse principale. Des implications pratiques pour la formation initiale des enseignants d'EPS sont évoquées.
\end{abstract}

Mots-clés : enseignement, domaines de valeurs, structure des valeurs, bien-être au travail, formation initiale.

Summary: The purpose of this paper is to analyze the relationship between Physical Education (PE) trainee teachers' values and their level of psychological well-being at work. The main working hypothesis is that PE's trainee teachers who valorize openness to change's and self-transcendence's orders values exhibit higher levels of psychological well-being at work. On the other hand, teachers who valorize conservation's values exhibit a lower one. An empirical survey was conducted among 184 trainee teachers in PE (58 women and 126 men) from 28 different universities in France. Results validate the main hypothesis. The practical implications for PE training are envisaged.

Keywords: teaching, higher orders values, values'structure, well-being at work, initial training. 


\section{Introduction}

Les valeurs n'ont jamais disparu de l'éducation pour la simple raison qu' « il n'y a pas d'éducation sans valeurs » (Reboul, 2016, p. 1). Selon l'auteur (Reboul, 1992), les valeurs de l'éducation renvoient à ce qui unit. Dès lors, elles sont à la fois individuelles et collectives. Les résultats des études montrent que les valeurs diffèrent d'un individu à l'autre et sont la plupart du temps intériorisées (Schwartz, 2006). Or, les valeurs sont les principes directeurs des attitudes et des comportements (Schwartz, 1992). Elles permettent de les prédire (Schwartz et al., 2012).

Dans la formation des enseignants, aborder la notion de «valeurs » ne va pas de soi (Beitone et Hemdane, 2018). Ainsi, pour les fonctionnaires stagiaires, les valeurs à transmettre seraient floues (ibid.). Pourtant, l'enseignement est un métier généralement motivé par des valeurs (Sahlberg, 2010) et la formation initiale serait une étape importante dans la consolidation de celles-ci (Johnson et Elder, 2002), notamment en EPS (Curtner-Smith et al., 2008). En effet, la formation initiale participe à construire une «identité professionnelle et une "culture commune" quant aux valeurs» (Perrenoud, 1994, p. 211).

Rascle et Bergugnat (2013) soulignent le malaise prégnant chez les enseignants stagiaires. Seulement 10 à $15 \%$ des enseignants ne se plaignent pas dès leur entrée dans la profession. Cette forme de souffrance au travail influe directement sur la qualité de leur enseignement (Jeffrey et Sun, 2006). Selon Favre (2008), les valeurs favorisent «les motivations visant le développement psychologique » (p. 73). La littérature récente montre que certaines valeurs favorisent le bien-être. Dans la littérature anglaise, cette tendance a également été démontrée chez les enseignants (Sortheix et Schwartz, 2017). Cependant, les recherches n'interrogent pas si certaines valeurs sont plus propices au bien-être au travail, ni même si ces relations sont effectives dans l'enseignement en EPS. Il semble donc important de s'intéresser à ce milieu spécifique. En effet à l'instar de Bardi et Schwartz (2013), l'EPS se distinguerait dans la transmission des valeurs.

Il semble donc pertinent d'explorer les relations entre valeurs et bien-être au travail et plus précisément le bien-être psychologique au travail (BEPT) des enseignants stagiaires. L'objet du présent article est d'examiner ces relations et de déterminer si certaines valeurs sont plus propices au BEPT.

\section{Bien-être psychologique au travail et valeurs : éclairages théoriques}

\section{Le bien-être psychologique au travail}

Plusieurs travaux s'intéressent aux enseignants stagiaires (Rayou et Ria, 2009) et à leur bien-être (Rascle et Bergugnant, 2013). Les résultats révèlent que la formation préprofessionnelle est une période critique au niveau du bien-être ressenti (ibid.). Il s'agit toutefois du bien-être général et non du bien-être au travail (Kashdan et al., 2008), alors que la littérature récente défend la spécificité de ce dernier (Dagenais-Desmarais et Savoie, 2012). Ainsi, il semble essentiel d'interroger spécifiquement les liens entre le bien-être et les valeurs chez cette population spécifique d'enseignants stagiaires.

Le développement de la psychologie positive a modifié la manière d'interroger le bien-être (Dagenais-Desmarais et Savoie, 2012). Dans ce cadre, les travaux de Massé et ses collaborateurs (1998) ont développé l'approche complémentaire du bien-être. Elle est caractérisée par le bien-être psychologique et a été opérationnalisée dans le contexte professionnel par Dagenais-Desmarais et Savoie (2012). Elle interroge de manière concomitante les deux approches principales du bien-être : hédonique (Diener, 1984) et eudémonique (Waterman, 1993). Le bien-être hédonique correspond à vivre davantage d'émotions positives que négatives (Diener, 1984) tandis que le bien-être eudémonique représente la réalisation de soi (Waterman, 1993). Et si le bien-être hédonique est prédominant dans la littérature sur le bien-être, ce n'est pas le cas dans le domaine spécifique du bien-être au travail (Massé et al., 1998).

Dans la présente étude, le BEPT sera compris comme "une expérience subjective positive où l'on tend à exprimer le meilleur de soi et qui se construit à travers soi, à travers ses relations sociales au travail et dans les interactions avec son organisation » (Dagenais-Desmarais et Privé, 2010, p. 70). Selon Dagenais-Desmarais et Savoie (2012), le concept comprend cinq dimensions : "l'adéquation interpersonnelle au travail, l'épanouissement au travail, le sentiment de compétence au travail, la volonté d'engagement au travail et la reconnaissance perçue au travail » (p. 668 ; annexe 1). Le BEPT de l'enseignant se mesure au travers d'une approche globale par rapport à son environnement professionnel. 


\section{Le modèle théorique des valeurs humaines de base}

De nombreux auteurs ont cherché à définir les valeurs. Parmi ces nombreuses approches, nous avons choisi d'utiliser le cadre théorique de Schwartz (1992). La théorie des valeurs de Schwartz (1992; 2006) présente un modèle ayant une validité théorique et empirique dans différents domaines (Bardi et Schwarz, 2013). Selon Hitlin et Piliavin (2004), ce modèle est le plus répandu dans la littérature internationale. L'auteur considère les valeurs comme des principes directeurs qui permettent de faire des sélections ou des évaluations d'actions (Schwartz, 2006). La construction des valeurs est conditionnée par trois paramètres : «satisfaire les besoins biologiques des individus, permettre l'interaction sociale et assurer le bon fonctionnement et la survie des groupes » (ibid., p. 931).

Il présente une structure universelle de dix valeurs de base : l'autonomie (créativité, curiosité, liberté), la stimulation (audace, vie excitante et variée), l'hédonisme (avoir du bon temps dans la vie), la réussite (ambition, détermination), le pouvoir (autorité, richesse), la sécurité (sécurité nationale, ordre, propreté), la tradition (humilité, dévotion), la conformité (obéissance, politesse), la bienveillance (solidaire, altruisme) et l'universalisme (justice sociale, égalité, protection de l'environnement) (Figure 1). Ces dix valeurs sont regroupées en quatre domaines de valeurs (Schwartz, 2006) : l'ouverture au changement (indépendance, vivre de nouvelles expériences excitantes), l'affirmation de soi (poursuite d'intérêts individuels), la continuité (respect des normes sociales et des habitudes) et le dépassement de soi (être tourné vers les autres) (Figure 1). De plus, le modèle peut être actualisé en fonction de l'avancée des connaissances. Schwartz et ses collaborateurs ont enrichi la structure de base de 10 valeurs en arrivant à un découpage du circumplex en 19 valeurs de base, encore peu utilisé au niveau empirique (2012).

Dès lors, «les individus, comme les groupes, se distinguent par les priorités qu'ils donnent à ces valeurs » (ibid., p. 929). Ainsi, ces valeurs sont présentes chez chaque individu (universelles) de manière plus ou moins marquée. La hiérarchie personnelle des valeurs (Schwartz et al., 2012) compose le système de valeurs d'une personne. La figure 1 présente l'agencement des valeurs de base dans une structure circulaire (Schwartz, 1992).

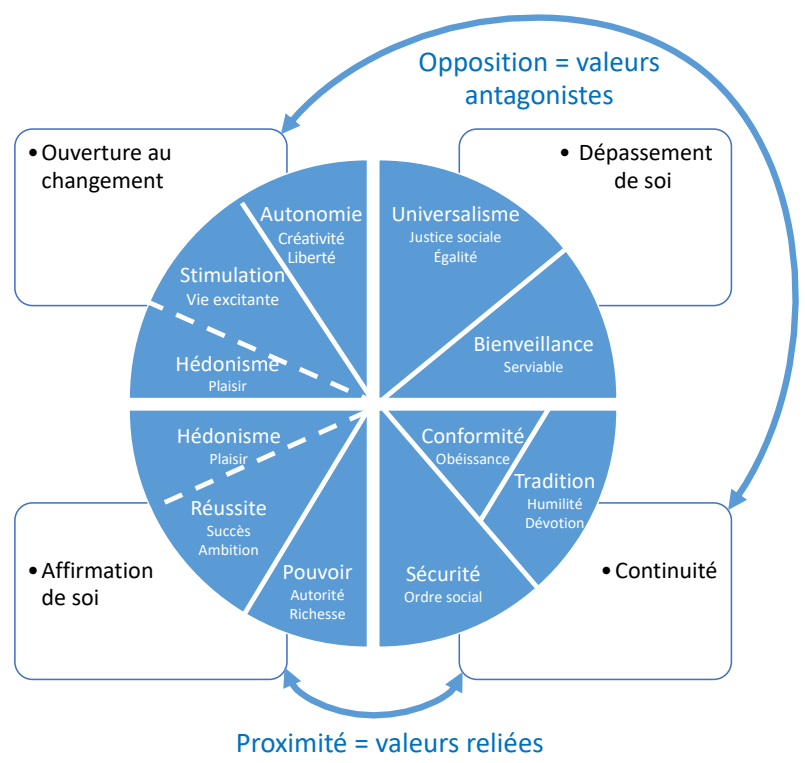

Figure 1. Le modèle théorique de Schwartz: le circumplex des dix valeurs humaines de base réparties en quatre dimensions de valeurs (inspiré de Schwartz, 2006)

Dans ce circumplex, la proximité ou l'éloignement visuel des valeurs traduit les relations de compatibilité ou d'antagonisme entre-elles. Les valeurs sont interdépendantes et s'influencent entre-elles. Les relations entre les valeurs sont dynamiques et fonctionnent comme un "vase communicant». Ainsi et pour exemple, la valeur de l'autonomie est opposée à la valeur sécurité mais reliée car adjacente à la valeur de stimulation.

Des études ont permis de valider le modèle empiriquement en France (Wach et Hammer, 2003a, b). Les auteurs ont participé à confirmer puis à diffuser ces résultats avec une présentation du modèle de Schwartz dans la littérature francophone (ibid.). Le cadre a été appliqué à différents domaines de la vie y compris le sport (Albouza, 2016 en France). Dans la littérature francophone, Morchain (2009) a souligné que les valeurs sont impactées par l'environnement social de l'individu. Toutefois, ce cadre est plutôt 
méconnu en France et peu de travaux le mobilisent dans l'enseignement (Albouza, 2016). À notre connaissance, il n'a jamais été utilisé pour des fonctionnaires stagiaires en EPS.

Knafo et Sagiv (2004) ont souligné les spécificités des valeurs selon les métiers ou groupes professionnels. La théorie des valeurs de Schwartz appliqué à l'éducation en France a permis l'élaboration d'un outil pédagogique pour l'orientation (Leyrit, 2013) puis pour l'orientation scolaire et professionnelle (Gros et Wach, 2013) avec des propositions d'applications. De plus, Gosling et Wach (2010) proposent une liste inspirée des valeurs de Schwartz dans un logiciel d'aide à l'orientation (le logiciel Valeurs). Les résultats montrent que les étudiants du même cursus ont des valeurs très proches comme l'avaient démontré Schwartz et ses collaborateurs (2012). Or, une certaine stabilité des valeurs une fois adulte est aussi révélée (ibid.). Dès lors, cela conforte l'importance de la formation initiale dans la construction des valeurs en milieu professionnel.

S'agissant des enseignants et des enseignants stagiaires, une étude (Schwartz et Bardi, 2001) dans 57 pays, dont la France, montre qu'ils sont davantage orientés vers l'autonomie et donc l'ouverture au changement comparativement à la population globale des 14 pays d'Europe (ibid.). Cette étude et d'autres montrent que plus le niveau d'éducation est développé, plus les valeurs de l'ouverture au changement sont valorisées et les valeurs de continuité dévalorisées (Wach et Hammer, 2003a ; Schwartz, 2006). L'augmentation de l'âge a un effet inverse : elle est corrélée positivement avec les valeurs de continuité et négativement avec l'ouverture au changement (ibid.). De plus, les femmes valorisent davantage les valeurs de continuité et de dépassement de soi que les hommes. À l'inverse, les hommes valorisent davantage l'affirmation de soi et l'ouverture au changement (Wach et Hammer, 2003a ; Schwartz et Rubel, 2005). Concernant ces variables socio-démographiques, il convient de souligner pour comprendre les différences de système de valeurs, que l'âge puis le niveau d'éducation sont plus déterminants que le sexe (Wach et Hammer, 2003a). Dès lors et contrairement aux idées reçues, le fait d'être un homme ou une femme différencie l'importance que les individus accordent aux valeurs (ibid.).

En revanche, aucune étude à notre connaissance n'interroge spécifiquement les systèmes de valeurs des enseignants d'EPS et notamment ceux des enseignants débutant dans le métier.

\section{Bien-être et valeurs}

Au niveau conceptuel, Sortheix et Schwartz (2017) montrent que les domaines de valeurs de l'ouverture au changement et du dépassement de soi sont basés sur la «croissance individuelle » par rapport à leurs bases motivationnelles plus intrinsèques et seraient protectrices pour l'individu. À l'inverse, les domaines de valeurs de l'affirmation de soi et de la continuité sont plutôt reliés à l'anxiété et des motivations plus extrinsèques. La valeur de réussite se retrouve dans les deux sources de motivation. Par ailleurs, les fondements du bien-être eudémonique (Waterman, 1993) développent l'idée que la réalisation de son plein potentiel peut être atteinte par la réalisation d'objectifs liés aux valeurs.

Des études ont montré des relations entre les valeurs de l'autonomie, la réussite, la stimulation, la bienveillance et l'universalisme et le bien-être hédonique (Sagiv et Schwartz, 2000). Sortheix et Schwartz (2017) soutiennent que l'ouverture au changement est corrélée positivement avec le bien-être hédonique alors que les valeurs de continuité y sont corrélées négativement (ibid.). Par ailleurs, Bojanowska et Piotrowski (2018) montrent que l'ouverture au changement, la continuité et le dépassement de soi sont liés à des scores plus élevés de bien-être eudémonique. L'ouverture au changement est un domaine de valeurs propice au bien-être dans ses différentes approches. Toutefois, ces résultats se différencient pour les autres domaines de valeurs; ils méritent donc d'être interrogés avec une approche complémentaire du bien-être, ce qui n'a jamais été fait à notre connaissance, pas plus que dans le contexte spécifique du bien-être au travail. Dès lors, aucune recherche n'a examiné le lien entre les valeurs et le bien-être psychologique au travail des enseignants.

\section{Objectif}

L'objet de notre travail est d'examiner le lien entre le système de valeurs des enseignants stagiaires en EPS et leur BEPT. L'hypothèse de ce travail étant que les enseignants stagiaires en EPS qui valorisent les valeurs d'ouverture au changement et de dépassement de soi ont un plus haut niveau de bien-être psychologique au travail. À l'inverse, ceux qui valorisent les valeurs de continuité et d'affirmation de soi ont un niveau de BEPT plus faible. 


\section{Méthode}

\section{Participants}

Cent quatre-vingt-quatre enseignants stagiaires (58 femmes et 126 hommes) en EPS en France ont participé à l'étude en mars 2018. L'échantillon représente $21 \%$ de la population ciblée, il s'agit des fonctionnaires admis au Certificat d'Aptitude au Professorat d'Éducation Physique et Sportive et au Certificat d'Aptitude aux Fonctions d'enseignement dans les Établissements Privé, non titularisés en mars 2018. L'échantillon est représentatif de la population cible en termes de genre et de statut (public/privé). Ces variables ont été contrôlées séparément avec un test de conformité du Khi-deux ( $p>0,2)$.

\section{Outil}

Les participants ont répondu à un questionnaire unique permettant de mesurer le système général de valeurs (partie 1) et le BEPT (partie 2). La durée de passation était d'environ 10 minutes. Des questions préliminaires permettaient également de recueillir les variables sociologiques.

\section{Système général de valeurs : le PVQ-21}

Le modèle théorique est principalement basé sur un questionnaire « de valeurs par portraits » (PVQ Schwartz et al., 2001) dans lequel les individus sont comparés à des «courts portraits » d'individus représentant les valeurs fondamentales de Schwartz. L'outil de mesure validé est le $P V Q-21$ de Schwartz (2003), il s'agit du plus répandu dans la recherche internationale sur les valeurs notamment en raison de son usage dans les enquêtes de l'enquête bi-annuelle de l'European Social Survey (Cieciuch et al., 2018). Ce questionnaire est composé de 21 items : entre quatre et six items par domaine de valeurs. Chaque item décrit brièvement le comportement d'une personne auquel il faut se référer. Les réponses se font à travers des échelles d'appréciation en six points (1: «pas du tout comme moi »; $6:$ «tout à fait comme moi »). Les scores par dimensions de valeurs seront privilégiés comme le préconisent les études sur le thème (e.g., Cieciuch et al., 2018). L'outil de mesure est proposé en annexe 2.

\section{Bien-être psychologique au travail : l'IBEPT}

L' «Index de Bien-être Psychologique au Travail » validé par Dagenais-Desmarais et Savoie (2012) a été largement utilisé dans le monde scientifique (Loup, 2016). C'est un questionnaire en 25 items regroupés dans les cinq dimensions du bien-être psychologique suscitées (5 items par dimension).

L'outil de mesure est proposé en annexe 1. Il est composé de plusieurs énoncés décrivant comment les gens peuvent se sentir au travail. Une échelle de type Lickert en 6 points (de 1: «En désaccord» à 6 : «Tout à fait en accord») permet aux répondants de se positionner pour évaluer leur IBEPT au cours des quatre dernières semaines. L'IBEPT se calcule au travers de scores par dimension ou/et avec un score total. Ce résultat est obtenu en calculant la moyenne des scores par dimension ou pour le questionnaire. Plus le score tend vers six, plus l'enseignant a un niveau de bien-être élevé.

\section{Procédure}

Un courrier électronique a été adressé aux responsables de la formation de master Métiers de l'Enseignement, de l'Éducation et de la Formation (MEEF), parcours EPS dans laquelle les enseignants stagiaires étaient inscrits en janvier 2018. L'objectif de ce premier courriel était d'avoir un accord de passation du questionnaire électronique auprès de la promotion d'enseignants stagiaires. Sur les 29 enseignants responsables contactés, un seul responsable n'a pas répondu favorablement à notre sollicitation. Celles et ceux ayant répondu favorablement ont ensuite été sollicités pour communiquer le questionnaire sur une période déterminée en mars et avril 2018 à l'ensemble de leurs étudiants fonctionnaires stagiaires.

Chaque responsable de formation a reçu des consignes précises formalisées par écrit. Elle permettait de présenter l'étude, d'apporter des précisions concernant l'échelle de réponse et enfin de rappeler le caractère anonyme du questionnaire.

L'étude a été déclarée à la correspondante Informatique et Libertés de notre Université sous le numéro 2140484. 


\section{Traitement et analyses}

Pour utiliser les données du questionnaire de valeurs par portrait, nous avons effectué la « correction d'échelle » préconisée par Schwartz (2003). Chaque score est centré par rapport à la moyenne de l'individu (Wach et Hammer, 2003a). Cette démarche convertit les scores en valeur positive et négative selon l'importance accordée à la valeur par rapport aux autres valeurs. Un score positif indiquera une prévalence pour un domaine de valeurs. Au contraire, un score négatif pour ce domaine révélera une faible importance de ce dernier dans le système de valeurs de l'individu. Ainsi, avec un score pour le domaine de la continuité de 5,0 alors que la moyenne des scores bruts de l'individu est de 4,2. La différence de $+0,8$ indique le niveau de prévalence du domaine de valeurs continuité. Pour l'IBEPT, un score global de BEPT a été calculé comme préconisé par Dagenais-Desmarais et Savoie (2012).

La normalité des données a été vérifiée avec un histogramme, une droite de Henry et le test de Kolmogorov-Smirnov. Des méthodes statistiques standards ont permis de calculer les scores centrés, les moyennes et les écarts-types de chaque variable. Les données ont été traitées avec le logiciel $\mathrm{R}$ studio (version 1.2.13.35) pour l'analyse des corrélations de Pearson. Le seuil de significativité a été fixé à $\mathrm{p}=$ 0,05 .

\section{Résultats}

\section{Une hiérarchie de valeurs identique pour les enseignants stagiaires en EPS}

Au niveau descriptif, le tableau 1 montre les moyennes et les écarts-types par domaine de valeurs pour les enseignants stagiaires en EPS.

Tableau 1. Statistiques descriptives des domaines de valeurs pour les enseignants stagiaires et pour les données de l'ESS8 dans le contexte français.

\begin{tabular}{ccccc} 
& Dépassement de soi & $\begin{array}{c}\text { Ouverture au } \\
\text { changement }\end{array}$ & Affirmation de soi & Conservation \\
\hline Stagiaire EPS & $0,95 \pm 0,42$ & $0,29 \pm 0,41$ & $-0,53 \pm 36$ & $-0,32 \pm 0,39$
\end{tabular}

Les résultats montrent que la hiérarchie entre les domaines de valeurs est identique pour les enseignants stagiaires en EPS comparativement à la littérature en langue anglaise. Ils valorisent en priorité le dépassement de soi puis l'ouverture au changement; la continuité et enfin l'affirmation de soi.

\section{Valoriser les valeurs de dépassement de soi et d'ouverture au changement pour être en bien-être psychologique au travail.}

Tous enseignants confondus, l'indice de bien-être psychologique au travail est de 4,74 $\pm 0,64$. Le tableau 2 présente les corrélations entre les domaines de valeurs et l'indice de bien-être psychologique chez les enseignants stagiaires en EPS.

Tableau 2. Corrélation entre l'indice de bien-être psychologique au travail et les scores du PVQ par dimension de valeurs

\begin{tabular}{lllll} 
& $\begin{array}{l}\text { Ouverture au } \\
\text { changement }\end{array}$ & $\begin{array}{l}\text { Dépassement } \\
\text { de soi }\end{array}$ & Continuité & $\begin{array}{l}\text { Affirmation } \\
\text { de soi }\end{array}$ \\
\hline $\begin{array}{l}\text { Bien-être } \\
\text { psychologique au } \\
\text { travail }\end{array}$ & $0,30^{* *}$ &, $21^{*}$ & $-0,24^{* *}$ & $-0,19$. \\
$\begin{array}{l}\text { Intervalle de } \\
\text { confiance à } 95 \%\end{array}$ & $0,16 / 0,42$ & $0,10 / 0,33$ & $-0.36 /-0,11$ & $-0,32 /-0,08$
\end{tabular}

\footnotetext{
$.=0,05<\mathrm{p}<0,10$

$*=\mathrm{p}<0,05$

$* *=p<0,001$
} 
Les résultats montrent que le domaine de l'ouverture au changement est le mieux corrélé avec un score élevé à l'IBEPT $(\mathrm{r}=0,30 ; \mathrm{p}<0,01)$. La relation avec le dépassement de soi révèle une corrélation moins puissante $(\mathrm{r}=0,21$ pour le dépassement de soi $; \mathrm{p}<0,05)$. Au contraire, les valeurs de continuité sont inversement corrélées au bien-être au travail des enseignants stagiaires $(r=-0,24 ; p<0,01)$. Avec un risque d'erreur supérieure, les valeurs d'affirmation sont négativement corrélées au BEPT $(r=-0,19$; $\mathrm{p}<0,05)$.

\section{Discussion}

L'objet de l'étude était de montrer le lien entre le système de valeurs des enseignants stagiaires en EPS et leur BEPT. Les résultats confirment l'hypothèse de ce travail: les valeurs d'ouverture au changement et de dépassement de soi sont positivement corrélées au BEPT pour les enseignants stagiaires en EPS. La corrélation est négative pour les valeurs de continuité. Par ailleurs, les enseignants d'EPS stagiaires valorisent davantage l'ouverture au changement comparativement à la population française en général.

\section{Le rôle de la formation pour développer les valeurs propices au BEPT des enseignants stagiaires en EPS}

Les résultats révèlent l'existence de relations directes entre les valeurs des enseignants stagiaires en EPS et le BEPT. Ces résultats renforcent ceux mentionnés dans la littérature anglaise (e.g., Sortheix et Schwartz, 2017) sur les relations entre les valeurs de base et le bien-être hédonique et eudémonique. Ainsi, le fait qu'un enseignant stagiaire d'EPS soit en mieux-être s'il adopte des pratiques dans lesquelles il est stimulé, autonome et tourné vers les autres, quel que soit le contexte d'enseignement, est une vraie plus-value pour les connaissances sur les relations entre les valeurs et le BEPT.

De nombreuses études montrent que les valeurs évoluent en fonction des expériences vécues (e.g., Bardi et Schwartz, 2013). Ces travaux soulignent l'importance de la formation initiale dans la consolidation des valeurs des enseignants au travail (Curtner-Smith et al., 2008). Par ailleurs, selon Goyette et Martineau (2018), un des objectifs clés de la formation initiale des enseignants est de favoriser le développement d'une identité professionnelle lié au bien-être avec une réflexion sur soi. Les résultats de la présente étude préconisent de valoriser les valeurs de coopération par des groupes de pratique dans un environnement créatif, autonome et stimulant dans la formation initiale des professeurs d'EPS afin de faciliter l'entrée dans le métier.

Ce résultat est particulièrement intéressant au regard des résultats de Ochoa et Blanch (2018) qui ont montré l'importance d'investir le champ du BEPT pour viser la performance, l'efficience, la qualité, la compétitivité et le climat de travail. Ceci paraît d'autant plus essentiel que le bien-être des enseignants impacte celui des élèves (Rascle et Bergugnat, 2016). Par ailleurs, Bardi et ses collaborateurs (2014) ont montré que l'augmentation dans l'importance d'une valeur amène une diminution dans l'importance d'une valeur contradictoire. Dès lors, il nous paraît essentiel de limiter l'utilisation des valeurs d'ambition et d'autorité (affirmation de soi) déjà particulièrement développées chez les enseignants stagiaires en EPS au profit des valeurs d'entraide et d'indulgence (dépassement de soi) dans la formation initiale. Ainsi, supprimer les notes en master 2 MEEF-EPS et favoriser un fonctionnement collaboratif entre les fonctionnaires stagiaires pour les mémoires de recherche seraient une évolution possible basée sur les résultats de cette recherche. Ces travaux pourront être poursuivis dans d'autres disciplines d'enseignement. Il est d'autant plus important de faciliter le bien-être au travail des enseignants stagiaires qui sont particulièrement en souffrance au travail (Rascle et Bergugnat, 2013). Toutefois, cette étude n'interroge pas les spécificités de l'environnement de travail de chaque enseignant à l'instar d'autres paradigmes (psychologie du travail, didactique, ergonomie, etc.).

\section{Conclusion}

L'objet de l'étude était d'étudier la relation des valeurs sur le bien-être psychologique au travail des enseignants stagiaires en EPS.

Les résultats ont montré que les valeurs d'ouverture au changement et du dépassement de soi sont propices au BEPT des enseignants stagiaires en EPS. À l'inverse, les valeurs de continuité sont défavorables au bien-être au travail. Cette thématique mérite une attention scientifique particulière puisque les valeurs sont liées au BEPT des enseignants stagiaires en EPS et par voie de conséquence 
impactent également le bien-être des élèves à l'école (Rascle et Bergugnat, 2016); le tout participant à un meilleur enseignement et un meilleur apprentissage (Skaalvik et Skaalvik, 2011).

\section{Bibliographie}

Albouza, Y., Wach, M., Chazaud, P., \& Crémieux, J. (2016). Les valeurs des jeunes boxeurs au crible du modèle de Schwartz. Staps, 111(1), 97.

Bardi, A., Buchanan, K. E., Goodwin, R., Slabu, L., \& Robinson, M. (2014). Value stability and change during self-chosen life transitions: Self-selection versus socialization effects. Journal of Personality and Social Psychology, 106(1), 131-147.

Bardi, A., \& Schwartz, S. H. (2013). How does the value structure underlie value conflict. In J. Whitehead (Éd.), Values in youth sport and physical education. London : Routledge, pp. 137-151.

Beitone, A., \& Hemdane, E. (2018). École, savoirs et valeurs: Une tentative d'éclaircissement. Éducation et socialisation. Les Cahiers du CERFEE, Online, 48.

Bergugnat, L., \& Rascle, N. (2013). Les risques professionnels pour les enseignants débutants : Quelles ressources pour leur entrée dans le métier? Revue d'Épidémiologie et de Santé Publique, 61, 199-344.

Biétry, F., \& Creusier, J. (2013). Proposition d'une échelle de mesure positive du bien-être au travail (EPBET). Revue de gestion des ressources humaines, 87(1), 23.

Bojanowska, A., \& Piotrowski, K. (2018). Two levels of personality: Temperament and values and their effects on hedonic and eudaimonic well-being. Current Psychology, Online, 1-9.

Cieciuch, J., Davidov, E., Algesheimer, R., \& Schmidt, P. (2018). Testing for Approximate Measurement Invariance of Human Values in the European Social Survey. Sociological Methods \& Research, 47(4), 665-686.

Curtner-Smith, M. D., Hastie, P. A., \& Kinchin, G. D. (2008). Influence of occupational socialization on beginning teachers'interpretation and delivery of sport education. Sport, Education and Society, 13(1), 97-117.

Dagenais-Desmarais, V., \& Privé, C. (2010). Comment améliorer le bien-être psychologique au travail? Gestion, 35(3), 69.

Dagenais-Desmarais, V., \& Savoie, A. (2012). What is psychological well-being, really? A grassroots approach from the organizational sciences. Journal of Happiness Studies, 13(4), 659-684.

Diener, E. (1984). Subjective well-being. Psychological Bulletin, 95(3), 542-575

Favre, D. (2008). Chapitre 5 : Valeurs et motivation: Comment les valeurs pourraient constituer des obstacles à l'évolution des pratiques pédagogiques? In Favre D., Hasni A. (dir.). Les valeurs explicites et implicites dans la formation des enseignants. Bruxelles : De Boeck Supérieur, pp. 73-89.

Gosling, P., \& Wach, M. (2010). Le logiciel Valeurs. Pontoise : Éditions Delta Expert.

Goyette, N., \& Martineau, S. (2018). Les défis de la formation initiale des enseignants et le développement d'une identité professionnelle favorisant le bien-être. Phronesis, 7(4), 4-19.

Gros, M.-H., \& Wach, M. (2013). Intérêt des valeurs, valeurs et intérêts dans un bilan d'orientation. L'Orientation scolaire et professionnelle, Online, 42/3.

Hitlin, S., \& Piliavin, J. A. (2004). Values : Reviving a dormant concept. Annual Review of Sociology, 30(1), 359-393.

Jeffrey, D., \& Sun, F. (2006). Enseignants dans la violence. Québec : Presses de l’Université Laval.

Johnson, M. K., \& Elder, G. H. (2002). Educational Pathways and Work Value Trajectories. Sociological Perspectives, 45(2), 113-138.

Kashdan, T. B., Biswas-Diener, R., \& King, L. A. (2008). Reconsidering happiness : The costs of distinguishing between hedonics and eudaimonia. The Journal of Positive Psychology, 3(4), 219-233.

Knafo, A., \& Sagiv, L. (2004). Values and work environment: Mapping 32 occupations. European Journal of Psychology of Education, 19(3), 255-273.

Leyrit, H. (2013). L'inventaire des valeurs de Schwartz: Un outil pédagogique pour l'orientation. Présenté à Sherbrooke. Sherbrooke: VIIe Colloque des Questions de Pédagogie dans l'Enseignement Supérieur.

Loup, P. (2016). Influence des technologies nomades sur le bien-être au travail : Une lecture par la théorie de la conservation des ressources (Doctoral dissertation). Université Montpellier.

Massé, R., Poulin, C., Dassa, C., Lambert, J., Bélair, S., \& Battaglini, M. A. (1998). Élaboration et validation d'un outil de mesure du bien-être psychologique : L'ÉMMBEP. Canadian Journal of Public Health, 89(5), 352-357.

Morchain, P. (2009). Psychologie sociale des valeurs. Malakoff : Dunod. 
Ochoa, P., \& Blanch, J. (2018). Psychosocial well-being at work: Reasons to invest in healthy employees and workplaces. In P. Ochoa, M. T. Lepeley, \& P. Essens, Wellbeing for sustainability in the global workplace. Londres : Routledge, pp. 10-23.

Perrenoud, P. (1994). La formation des enseignants entre théorie et pratique. Paris : L'Harmattan.

Rascle, N., \& Bergugnat, L. (2016). Qualité de vie des enseignants en relation avec celle des élèves: Revue de question, recommandations. Consulté à l'adresse CNESCO : $\quad$ http://www.cnesco.fr/wpcontent/uploads/2017/10/170929_enseignants.pdf.

Rayou, P., \& Ria, L. (2009). Former les nouveaux enseignants. Autour des statuts, de l'organisation et des savoirs professionnels. Éducation et sociétés, 23(1), 79.

Reboul, O. (1992). Les valeurs de l'éducation. Paris : Presses Universitaires de France.

Reboul, O. (2016). La philosophie de l'éducation. Paris : Presses Universitaires de France.

Reis, H. T., Sheldon, K. M., Gable, S. L., Roscoe, J., \& Ryan, R. M. (2000). Daily well-being: The role of autonomy, competence, and relatedness. Personality and social psychology bulletin, 26(4), 419-435.

Sagiv, L., \& Schwartz, S. H. (2000). Value priorities and subjective well-being: Direct relations and congruity effects. European Journal of Social Psychology, 30(2), 177-198.

Schwartz, S. H. (1992). Universals in the Content and Structure of Values: Theoretical Advances and Empirical Tests in 20 Countries. In M. P. Zanna, Advances in Experimental Social Psychology San Diego : Academic Press, Vol. 25, pp. 1-65.

Schwartz, S. H. (2003). A proposal for measuring value orientations across nations. Questionnaire Package of the European Social Survey, Online, 259-290.

Schwartz, S. H. (2006). Les valeurs de base de la personne: Théorie, mesures et applications. Revue française de sociologie, 47(4), 929.

Schwartz, S. H., \& Bardi, A. (2001). Value Hierarchies Across Cultures : Taking a Similarities Perspective. Journal of Cross-Cultural Psychology, 32(3), 268-290.

Schwartz, S. H., Cieciuch, J., Vecchione, M., Davidov, E., Fischer, R., Beierlein, C., Ramos, A., Verkasalo, M., Lönnqvist, J. E., Demirutku, K., Dirilen-Gumus, O. \& Konty, M. (2012). Refining the theory of basic individual values. Journal of Personality and Social Psychology, 103(4), 663-688.

Schwartz, S. H., Melech, G., Lehmann, A., Burgess, S., Harris, M., \& Owens, V. (2001). Extending the crosscultural validity of the theory of basic human values with a different method of measurement. Journal of CrossCultural Psychology, 32(5), 519-542.

Skaalvik, E. M., \& Skaalvik, S. (2011). Teacher job satisfaction and motivation to leave the teaching profession : Relations with school context, feeling of belonging, and emotional exhaustion. Teaching and Teacher Education, 27(6), 1029-1038.

Sortheix, F. M., \& Schwartz, S. H. (2017). Values that underlie and undermine well-being: Variability across countries: values that underlie and undermine well-being. European Journal of Personality, 31(2), 187-201.

Wach, M., \& Hammer, B. (2003a). La structure des valeurs est-elle universelle? Genèse et validation du modèle compréhensif de Schwartz. Paris : L'Harmattan.

Wach, M., \& Hammer, B. (2003b). La structure des valeurs en France d'après le modèle de Schwartz. Revue internationale de psychologie sociale, 16(4), 47-85.

\section{Annexe}

Annexe 1 Indice de bien-être psychologique au travail

Ce questionnaire présente une liste d'énoncés décrivant comment les gens peuvent se sentir au travail. En considérant votre travail au cours des quatre dernières semaines.

\begin{tabular}{|c|c|c|c|c|c|}
\hline En désaccord & $\begin{array}{c}\text { Très faiblement } \\
\text { en accord }\end{array}$ & $\begin{array}{c}\text { Faiblement en } \\
\text { accord }\end{array}$ & $\begin{array}{c}\text { Modéremment en } \\
\text { accord }\end{array}$ & En accord & $\begin{array}{c}\text { Tout à fait en } \\
\text { accord }\end{array}$ \\
\hline 1 & 2 & 3 & 4 & 5 & 6 \\
\hline
\end{tabular}

- Il n’y a ni bonnes ni mauvaises réponses

- Soyez simplement le plus sincère possible dans vos réponses.

- Toutes les réponses sont anonymes et confidentielles.

Veuillez indiquer à quel point vous êtes en accord avec chaque énoncé ? 


\begin{tabular}{|c|c|c|c|c|c|c|c|}
\hline 1 & J'apprécie les gens avec qui je travaille. & 1 & 2 & 3 & 4 & 5 & 6 \\
\hline 2 & Je trouve mon travail excitant. & 1 & 2 & 3 & 4 & 5 & 6 \\
\hline 3 & Je sais que je suis capable de faire mon travail. & 1 & 2 & 3 & 4 & 5 & 6 \\
\hline 4 & Je sens que mon travail est reconnu. & 1 & 2 & 3 & 4 & 5 & 6 \\
\hline 5 & J'ai envie de prendre des initiatives dans mon travail. & 1 & 2 & 3 & 4 & 5 & 6 \\
\hline 6 & Je trouve agréable de travailler avec les gens de mon travail. & 1 & 2 & 3 & 4 & 5 & 6 \\
\hline 7 & J'aime mon travail. & 1 & 2 & 3 & 4 & 5 & 6 \\
\hline 8 & J'ai confiance en moi au travail. & 1 & 2 & 3 & 4 & 5 & 6 \\
\hline 9 & Je sens que mes efforts au travail sont appréciés. & 1 & 2 & 3 & 4 & 5 & 6 \\
\hline 10 & Je me soucie du bon fonctionnement de mon organisation. & 1 & 2 & 3 & 4 & 5 & 6 \\
\hline 11 & Je m'entends bien avec les gens à mon travail. & 1 & 2 & 3 & 4 & 5 & 6 \\
\hline 12 & Je suis fier de l'emploi que j'occupe. & 1 & 2 & 3 & 4 & 5 & 6 \\
\hline 13 & Je me sens efficace et compétent dans mon travail. & 1 & 2 & 3 & 4 & 5 & 6 \\
\hline 14 & Je sais que les gens croient aux projets sur lesquels je travaille. & 1 & 2 & 3 & 4 & 5 & 6 \\
\hline 15 & J'aime relever des défis dans mon travail. & 1 & 2 & 3 & 4 & 5 & 6 \\
\hline 16 & J'ai une relation de confiance avec les gens de mon travail. & 1 & 2 & 3 & 4 & 5 & 6 \\
\hline 17 & Je trouve un sens à mon travail. & 1 & 2 & 3 & 4 & 5 & 6 \\
\hline 18 & J'ai le sentiment de savoir quoi faire dans mon travail. & 1 & 2 & 3 & 4 & 5 & 6 \\
\hline 19 & $\begin{array}{l}\text { J'ai l'impression que les gens avec qui je travaille reconnaissent ma } \\
\text { compétence. }\end{array}$ & 1 & 2 & 3 & 4 & 5 & 6 \\
\hline 20 & Je désire contribuer à l'atteinte des objectifs de mon organisation. & 1 & 2 & 3 & 4 & 5 & 6 \\
\hline 21 & Je me sens accepté comme je suis par les gens avec qui je travaille. & 1 & 2 & 3 & 4 & 5 & 6 \\
\hline 22 & J'ai un grand sentiment d'accomplissement au travail. & 1 & 2 & 3 & 4 & 5 & 6 \\
\hline 23 & Je connais ma valeur comme travailleur. & 1 & 2 & 3 & 4 & 5 & 6 \\
\hline 24 & Je sens que je suis un membre à part entière de mon organisation. & 1 & 2 & 3 & 4 & 5 & 6 \\
\hline 25 & $\begin{array}{l}\text { J'ai envie de m'impliquer dans mon organisation au-delà de ma charge de } \\
\text { travail. }\end{array}$ & 1 & 2 & 3 & 4 & 5 & 6 \\
\hline
\end{tabular}

Dimensions «Adéquation interpersonnelle au travail » : items 1, 6, 11, 16 et 21

Dimension «Épanouissement dans le travail »: items 2, 7, 12, 17 et 22

Dimensions «Sentiment de compétence au travail »: items 3, 8, 13, 18 et 23

Dimensions « Reconnaissance perçue au travail » : items 4, 9, 14, 19 et 24

Valeurs «Volonté d'engagement au travail »: items 5, 10, 15, 20 et 25

Annexe 2: Questionnaire de valeurs par portrait ${ }^{1}$ :

Les phrases qui suivent décrivent des personnes différentes. Veuillez lire chaque description et indiquer à quel point cette personne est ou n'est pas comme vous. Mettez un X dans la case à droite qui montre à quel point la personne décrite vous ressemble. Entoure un seul chiffre, c'est à dire celui qui correspond mieux à tes actions du quotidien

\begin{tabular}{|c|c|c|c|c|c|}
\hline $\begin{array}{c}\text { Pas du tout } \\
\text { comme moi }\end{array}$ & Pas comme moi & $\begin{array}{c}\text { Plutôt pas comme } \\
\text { moi }\end{array}$ & $\begin{array}{c}\text { Plutôt comme } \\
\text { moi }\end{array}$ & Comme moi & $\begin{array}{c}\text { Tout à fait } \\
\text { comme moi }\end{array}$ \\
\hline 1 & 2 & 3 & 4 & 5 & 6 \\
\hline
\end{tabular}

- Il n'y a ni bonnes ni mauvaises réponses

- Soyez simplement le plus sincère possible dans vos réponses.

- Toutes les réponses sont anonymes et confidentielles.

\footnotetext{
${ }^{1}$ Attention, le questionnaire est à différencier en fonction du sexe du répondant pour qu'il puisse pleinement s'identifier à la comparaison
} 
Dans quelle mesure cette personne vous ressemble?

1 Réfléchir à de nouvelles idées et être créatif c'est important pour lui/elle. Il/elle 1 aime faire les choses à sa manière.

2 C'est important pour lui/elle d'être riche. Il/elle veut avoir beaucoup d'argent e

2 des choses chères.

Il/elle pense que c'est important que tout être humain soit traité de manière

3 égale. Il/elle pense que tout le monde devrait avoir des chances égales dans la 1 vie.

4 C'est important pour lui/elle de montrer de quoi il/elle est capable. Il/elle veut

4 que les gens admirent ce qu'il/elle fait.

\begin{tabular}{|l|l|l|l|l|l|l|l|l|l}
\hline 5 & $\begin{array}{l}\text { Etre humble et modeste est important pour lui/elle. Il/elle essaie de ne pas } \\
\text { attirer l'attention sur lui/elle. }\end{array}$ & 2 & 3 & 4 & 5 & 6
\end{tabular}

Il/elle aime les surprises et recherche toujours de nouvelles choses à faire.

6 Il/elle pense que c'est important de faire beaucoup de choses différentes dans 1 la vie.

7 Vivre dans un environnement sécurisant est important pour lui/elle. Il/elle

évite tout ce qui Plaisir peut mettre en danger sa sécurité.

C'est important pour lui/elle d'écouter les gens qui sont différents de lui/elle

8 Même quand il/elle n'est pas d'accord avec eux, i1/elle tient quand même à les 1 comprendre.

Il/elle pense que les gens devraient faire ce qu'on leur dit. Il/elle pense que les

9 gens devraient toujours suivre les règles établies, même si personne ne les 1 surveille.

10 Avoir du bon temps est important pour lui/elle. Il/elle aime bien se faire plaisir.

11 C'est important pour lui/elle de décider tout seul de ce qu'il/elle va faire. Il/elle

1 aime être libre et ne pas dépendre des autres.

12 C'est très important pour lui/elle d'aider les gens qui l'entourent. Il/elle désire

se préoccuper de leur bien-être.

13 Avoir beaucoup de succès est important pour lui/elle. Il/elle espère que les 1

gens reconnaîtront ce qu'il/elle a fait.

\begin{tabular}{|l|l|l|l|l|l|l|}
\hline 14 & $\begin{array}{l}\text { Les traditions sont importantes pour lui/elle. Il/elle essaie de suivre les } \\
\text { coutumes transmises par la religion et la famille. }\end{array}$ & 2 & 3 & 4 & 5 & 6 \\
\hline
\end{tabular}

15 Il/elle recherche l'aventure et aime prendre des risques. Il/elle désire une vie excitante.

16 C'est important pour lui/elle que le gouvernement garantisse sa sécurité. Il/elle veut un État fort qui défende les citoyens. \begin{tabular}{|l|l|l|l|l|l|l|}
\hline 17 & $\begin{array}{l}\text { C'est important pour lui/elle d'être respecté par les autres. Il/elle veut que les } \\
\text { autres fassent ce qu'il/elle leur dit. }\end{array}$ & 2 & 3 & 4 & 5 & 6 \\
\hline
\end{tabular}

18 \begin{tabular}{l|l|l|l|l|l|l|l|}
$\begin{array}{l}\text { Être fidèle à ses amis est important pour lui/elle. Il/elle veut se dévouer pour } \\
\text { les personnes qui lui sont proches. }\end{array}$ & 1 & 2 & 3 & 4 & 5 & 6
\end{tabular}

19 \begin{tabular}{l|l|l|l|l|l|l} 
Il/elle pense que les gens doivent se soucier de la nature. S'occuper de \\
l'environnement est important pour lui.
\end{tabular}

20 \begin{tabular}{l}
$\begin{array}{l}\text { Se comporter toujours comme il faut est important pour lui/elle. Il/elle ne veut } \\
\text { rien faire que les gens puissent lui reprocher. }\end{array}$ \\
\hline
\end{tabular}

II/elle recherche toutes les occasions pour s'amuser. C'est important pour ${ }_{1}$

1 lui/elle de faire des choses qui lui procurent du plaisir.

Dimensions " Ouverture au changement» : items 1 et 11 (autonomie) + items 6 et 15 (stimulation)

Dimension «Affirmation de soi » : items 4 et 13 (réussite) + items 2 et 17 (pouvoir)

Dimensions «Continuité »: items 7 et 16 (conformité) + items 9 et 20 (tradition) + items 5 et 14

(sécurité)

Dimensions « Dépassement de soi » : items 12 et 18 (bienveillance) + items 3, 8 et 19 (universalisme)

Valeurs « Hédonisme » : items 10 et 21 